\title{
A Review on Intelligent Fault Detection in Rolling Element Bearings
}

\author{
Robin Raj Balraj ${ }^{1, *}$, Madhavi Barla $^{I}$ and Govardhan Tingarikar ${ }^{l}$ \\ ${ }^{1}$ ICFAI Foundation for Higher Education, Faculty of Science and Technology, Hyderabad, India
}

\begin{abstract}
Rolling element bearings play vital role in the working of rotating hardware or machine. The imperfection-initiated vibration signal estimation and its examination is frequently utilized in deficiency recognition of direction. The crude sign is mind boggling in nature to dissect for deformity highlights, Therefore the sign be prepared to break down it. This article presents different sign handling procedures including canny strategies, for example, Artificial Techniques, Machine learning techniques and so on. The suitability of these strategies, all things considered, depends on the idea of features isolated from the bearing signs. The writing containing procedures utilized by different analysts have been managed in this review. This review may fill in as a kind of perspective for the scientists to go over different strategies in bearing diagnostics.
\end{abstract}

\section{Introduction}

Rolling element bearings (REBs) are the most wellknown machine components playing an extraordinarily crucial job in practically a wide range of turning hardware and as such utilized finally in businesses due to their generally lower cost and operational straightforwardness. The move of turning apparatus is completely needy upon the wellbeing condition of the REBs, which represents nearly $50 \%$ of these gear disappointments [1-3]. The bearing shortcomings for example, spalling, stripping or sub case weariness because of misalignment, shaft incline, surface unpleasantness, high degree of waviness and considerations, and so on causes a breakdown of the framework which decreases the unwavering quality and accessibility of the plant [4]. An essential to actualize and grow compelling upkeep methodologies to limit the effect of disappointments because of failing of the moving component direction. The writing partitions the upkeep procedures basically into three sorts Corrective upkeep, Preventive support and Predictive upkeep three (or on the other hand Condition based maintenance (CBM). Huang et al. introduced a plain correlation of the different upkeep procedures and affirmed that the personal time and the all-inclusive harms are insignificant in CBM [5,6]. CBM is a dynamic procedure, which plans to evade sudden disastrous disappointments by early identification of early stage blames and actualizing the essential support activities for their seclusion, along these lines endeavouring to restore the strength of a machine, segment or framework. As of late, Shin and Jun looked into the assorted meanings of $\mathrm{CBM}$ and introduced another meaning of CBM as an upkeep arrangement that do support activity previously item disappointments occur, by evaluating item condition including working situations, and foreseeing the danger of item disappointments in an on-going manner, in light of accumulated item information [7]. In expansion, it has been expressed that the term CBM is frequently alluded to as Prognostics and Health Management (PHM). Signal preparing strategies used for the shortcoming analysis in mechanical frameworks have been accounted for in the writing. Howard checked on the different sign preparing methods for the discovery, analysis and guess of REBs up to the year 1994 [8]. Tandon and Chaudhary introduced the sign preparing approaches applied to the vibration furthermore, acoustic outflow signals for recognizable proof of deformities in REBs [9]. Jardine et al. gave an outline of the information obtaining, information preparing, finding and prognostics calculations actualized in the CBM of machines [10]. The writing clarified the wear instruments; wear checking and analysis techniques in REBs $[11,12]$. Greater part of the sign preparing strategies referred to into these papers are standard in nature and have been altered or utilized in blend with certain propelled signal preparing strategies in the course of the most recent fifteen years to improve their effectiveness and precision. The most punctual chips away at signal investigation to give a foundation to the scientists and further spotlights on the progressive updating in the sign handling strategies, which have been applied so far for the conclusion of issues in REBs.

This paper presents a concise rundown of the condition checking strategies applied to moving component. It also examines the contrast among finding and guess notwithstanding the pretended by include

"Corresponding author: robinraj335@gmail.com 
extraction forms in finding and forecast of moving component orientation; The articles related to flag handling strategies have been looked into and examined.

\section{Condition Monitoring Techniques}

Petersen et al. clarified this by saying that, "used the vibrations delivered in oneself modifying metal balls on gearbox and fan test devices to explore the store courses and assortments in robustness in view of value of flaws in the metal rollers" [13]. Patel et al. clarified this by saying that "the imitated and test vibration estimations to look at the effect of various close by flaws on the vibrations created by the moving part bearing" [14]. Saruhan et al. clarified this by saying that "coordinated vibration focuses on the moving part direction with internal race distortion, outside race disfigurement, ball flaw and a mix of the three under different weight and speed blends" [15]. Jena and Panigrahi, "developed a test set up to get the bearing vibration banners furthermore, rehearsed it to measure the width of the deformations seeded in the internal and outer races" [16]. The composing gives some all the more understanding into the employments of vibration condition checking as for moving segment bearing [17-21]. The sign getting ready systems inspected in the zone have been used to the vibration signals conveyed by the moving segment heading. Another generally used condition watching system is the acoustic release, which has been represented in the articles [22-26]. Pandya et al. clarified this by saying that, "the AE signals with Hilbert Huang change and used a K-nearest neighbour-based classifier for the assurance of issues in moving segment direction" [27]. Starting late, Chacon et al. assembled the AE signals from the hanging on for an outer race deformation and set it up with a wavelet-based sign planning procedure to perceive the bearing inadequacies in its starting periods, when the sign to upheaval extent is squat [28]. Starting late, a logically used conditionchecking gadget known as motor current imprint examination has gotten criticality in the weakness assurance of moving segment heading. Motor current imprint examination has Motor current mark investigation has been generally utilized in the territories where the defective parts are hard to access, for example, the divergent siphons covered in the grounds, atomic force plants, enlistment engine drives and so forth [2931]

\section{1. Intelligent Fault Diagnosis Techniques}

Diagnostics is the way toward exemplifying the status of harm through identification, separation what's more, recognizable proof utilizing the information gathered from the persistent wellbeing observing of the turning apparatus. Determination can be viewed as a backoccasion examination, which manages the shortcoming side effects in the machine and their arrangement. Jardine et al. expressed "machine issue finding as a As a Zhang et al. took care of the time and recurrence space measurable highlights extricated from the bearing informational collections to a gathering learning based steady bolster vector machine for the deficiency conclusion of moving component course [32-34]. The exhibition of these Artificial Intelligent (AI) methods depend to a huge degree on the distinction of highlights being utilized as contributions by them and henceforth the sign handling strategies must be proficient in the expulsion of issue qualities from the crude signs.

At first sign preparing strategies are related to course was extremely essential and fundamentally subordinate upon the check of certain verifiable boundaries, for instance, kurtosis, root mean square worth, mean, etc. Again, the imprint assessment procedures were not a lot of incredible in restricting the effect of uproars and impedance due to various wellsprings of vibration, for instance, riggings or as a result of the moving speed of the poles. Dunegan/Endevco's model 5712 early frustration discover was represented to distinguish the high repeat $\mathrm{AE}$ signals delivered by working orientation [35]. The analyser developed a sign above cutoff (SAT) (Scholastic Aptitude Test) estimation reliant on root mean square worth, open to high adequacy impacts of brief range. The analyser also had an envelope-perceived respect offer a continuously tricky hint of bearing condition. Cempel, made a ton of five discriminants for a stochastic vibro-acoustical technique top factor, drive factor, consonant factor, rice repeat, repeat change factor to isolate information about the ordinary procedure sufficiency, the repeat abundancy, ghost spread of the methodology, and time instabilities of the strategy range [36]. Cempel again settled a front line on the systems and procedures made and applied in vibroacoustieal (VA) diagnostics domain. He saw that, every lack makes its own VA Signal and described a great deal of indications both in the plentifulness and repeat territory of the VA signal $[37,38]$ the essayist has kept an eye on the issue of non-discontinuous inspiration train instigated in spalled heading. The autocorrelation and the Power spooky thickness of the model were then gained to think about the outcomes on the finding. Next, the essayist proposed two different ways to manage speak to the essentialness and repeat assortments due to nonperiodicity of the deformation drive train: (I) time-repeat typical for the load appointment (ii) showing the bearing by an immediate time-contrasting mass, spring, and damper system. Finally, the maker contemplated that the past is clear anyway doesn't depict the wonder totally; in separate, the latter is appropriate for the explanation, fortified by preliminary and numerical amusements. Pineyro et al. said that "the usage of three apparition strategies explicitly Second solicitation power supernatural thickness, Bispectral technique and Wavelet examination through Haar change on the bearing inadequacy signals, Second solicitation power ghost thickness prosperously reduced disturbance level and other tight band parts yet constrained a limitation since the unprecedented flimsy band resonances can't be perceived from irregular signs" [39]. The Bispectrum work was seen as sensitive to the recognizable proof of stage coupling tops in the range, counting those that are unquestionably not doubtlessly detectable in the power 
apparition thickness. Regardless, the hindrance is the measure of memory required during information handling. Wavelet techniques dependent on the Haar change showed to be use full in short transient recognition yet neglected to recognize drifters utilizing AE sensors.

In this portion, bit a couple of current signs taking care of procedures, for instance, wavelet change based strategies, Hilbert Huang change, morphological sign dealing with, etc have been discussed. Dron et al. coordinated assessments on a considerable lot of direction to test the distinctive parametric Autoregressive Spectrum examination techniques and complexity them and the common range (correlogram) assessment strategies [40]. The Autoregressive model close by Burg's figuring and Akaike information standard was held. The outcomes demonstrated that the parametric techniques have significant standards capacity and distinguish the defects early, whether or not the trademark frequencies are proximate to each other. Regardless, the Autoregressive strategies are flighty and must benefit the standard methods in lieu of superseding them. Altmann and Mathew, built up a novel procedure insinuated as 'envelope autoregressive range' for recognizing issue earnestness of low speed moving part direction $[41,42]$. The wavelet packs containing bearing issue related features were first removed from the discrete wavelet package assessment of vibration signals; second, a flexible framework predicated cushioned derivation structure executed on-line to choose wavelet bundles of interest lastly the various band-pass channel signal procured is utilized identified with the autoregressive (AR) scope of the envelope signal, to break down the bearing condition. Essential gains in the sign to-uproar extent are practiced and the method gives a responsive and precise reason to slant the bearing flaw fastidiousness. High repeat Acoustic Emission (AE) was utilized by Jamaludin and Mba to examine the prosperity state of amazingly moderate moving part direction using the Auto Regressive (AR) model of the bearing signs. It was known as consideration regarding that the $\mathrm{AE}$ is created due to the scouring action of roller on a hurt outside or internal race. Counting to the work bispectral signal examination methods clarified about in starting stage, further developed a watching strategy including a mix of sign planning, signal examination and modernized thinking procedures was finished by Yang et al.

\subsubsection{Spectral Kurtosis and Kurtogram:}

Lei et al. raised that "the Kurtogram reliant on the short period of time Fourier change (STFT) or FIR directs are not precise in the extraction of hasty features of a hurt bearing signature" [43]. An improved Kurtogram technique was propelled which took pleasure in the benefits of Daubechies-wavelet based Wavelet Packet Transform (WPT) channel. It was demonstrated to be ground-breaking in de-noising the signs and taking out the messed up features than the first kurtogram. It was computationally less troublesome similarly as quicker than the kurtogram reliant on the complex Morlet wavelet change. An improved frightful kurtosis method on bearing with outside and internal race insufficiencies was tried by, Wang and Liang [44-48] enhanced the Kurtogram scheme by swapping the temporal kurtosis of the signals extracted from the wavelet packet nodes as done in with the kurtosis of the corresponding power spectrum of their envelopes

\subsubsection{Cyclostationary methods:}

$\mathrm{He}$ and other worldly soundness hypothesis which was used by Ming et al. expected that the bearing flaw signals show proof of second request cyclostationarity and utilized it to plan an ideal Cyclic Wiener channel followed by an envelope examination of the separated sign by means of Hilbert change [49,50]. The cyclic birange being a three-dimensional dissemination and hard to speak to was cut along the cyclic recurrence hub ("called as once cut") and next the even cut ("along one recurrence pivot") of the once cut was practiced to analyse the bearing deficiencies. A few focal points, the cyclic otherworldly thickness, which Dong and Chen utilized is the Fourier change of the cyclic autocorrelation work got by adjusting the time changing autocorrelation capacity of a Wigner-Ville circulation dependent on the suspicion that the bearing signs have a cyclostationary character [51]. Wigner-Ville range so formed was then used to secure the time recurrence qualities of the vibration signals.

It was shown through imitated non-fixed signs that the methodology beats the Pseudo Wigner Ville scattering in controlling the deterrent terms and gives improved time repeat objectives than the Smoothed Pseudo Wigner Ville appointment similarly as it is computationally snappier than the other two. Thereafter, an assessment with the squared envelope go method on certifiable bearing issue signals mixed in with gear vibrations exhibited that the proposed approach is progressively viable in pulling out the bearing issue incorporates by suitably isolating the apparatus vibrations.

\subsubsection{Wavelet techniques:}

Introduced Presented by Wang et al. GHM multi-wavelet for better part extraction reinforced by an improved denoising plan reliant on a thresholding plan neighbouring coefficient subject to level (DLNeighCoeff). Multiwavelets offered the advantages of evenness, balance, limited assistance and high solicitation of transient minutes over the scalar wavelets [52]. The thresholding plan considered the effect of the neighbour coefficients and their dependence on different levels instead of thresholding term by term, along these lines giving practical camouflage of upheaval. Further, the assessment with observational mode breaking down, single worth decay, kurtogram and typical term-by-term wavelet denoising revealed that the new methodology defeats these strategies. Sawalhi and Randall [53-55]. The looking at makers thusly offered an adversary of symmetric certifiable Laplace wavelet (ARLW) channel that could be grown clearly from the bearing sign rather than choosing the wavelet limits. The Laplace wavelet was real and unfriendly to symmetric in nature, and best organized with the exponentially damped reaction 
capacity of the issue driving forces. Further the boundaries of the channel were updated by differential improvement computation using the kurtosis intensification rule. The results announced that the ARLW gives a dynamically deficient depiction of the sign and feasibly de-clatters the sign realizing a progression of the rash characteristics. The bearing deformation frequencies and the related music appear as spikes when found in the arranged envelope extend, it was seen by Dwindle and Wang. Taking into account this idea, they introduced another thought called as sparsogram for the bearing inadequacy area [56]. The sign was first crumbled utilizing the matched wavelet package change into arranged wavelet package center points at different breaking down levels and next the sparsity regards were figured from the power scope of the included wavelet group coefficients. Along these lines, sparsogram was created to pick the significant wavelet bundle center with moreover bearing weakness marks and sensible for the assurance of bearing issue conditions.

(D) Empirical mode decomposition:

Guo et al. communicated that "the fundamental edge mutilation and imperativeness spillage issues in waveletbased techniques" [57]. Further, the ordinary EMD (Electrical release machining) was represented to introduce mode mixing sway and the bowing of the flawed main impetuses. The related makers in this manner applied the EEMD (Ensemble Empirical Mode Decomposition) in view of the rule of EMD to the ghost kurtosis upgraded band pass filtered signal and procured natural mode capacities (IMFs). Later the IMF having the dominating relationship coefficient with the filtered signal was picked as the last sign freed from disturbance and arranged for extraction of the imperfect features. The proposed procedure handled the issue experienced by the standard EEMD in confining the bearing signs into the closeness of generous racket. Tsao et al. raised that "the EMD self-destructs the sign into different IMFs followed by the Hilbert run examination to reveal the repeat information concealed in the sign at any rate larger piece of the conveyances didn't have any principles for the game plan for fitting IMFs" [58,59]."

\section{(E) Matching Pursuit}

Coordinating interest calculation was used by Cui et al. despite a more defect arranged and flexible time repeat word reference containing the rotational repeat, blemish broadness, beat width and other drive information for the assessment of bearing imperfections. The proposed estimation handled the issue of redundancy of the word reference with additional points of interest of computationally speedier, consistent and controllable than the inherited count smoothed out planning interest, which uses the traditional, and new inspiration word references alluded to by the different makers. The composing depicted the section and leaves events of the moving segment bearing as a twofold impact ponders, transmitting step like and drive like responses. The related makers recently settled a quantitative relationship among the time opening between the two events besides, the inadequacy size followed by the development of another movement - drive word reference. Afterward, the new word reference was completely used with the genetic estimation updated planning enthusiasm to assess the spall size and expansion the precision of the distortion finding. It should be seen that this article has also added to the dedication made by Sawalhi and Randall and Zhao et al. in the quantitative finish of moving part heading $[60,61]$. A clear explanation of the planning interest count has been depicted in the reference [62]. Rai and Upadhyay introduced a through and cautious survey on signal preparing methods [63] [63]

\section{Conclusion}

The various signal processing techniques to overcome the challenges such as removal of back ground noise from vibration signal to extract the fault features with high resolution have been discussed. The methods to deal with stationary and non-stationary signals are discussed as well. The literature on difficulties in applying the techniques such as wavelet has been presented. This article would be useful for researchers to know and select appropriate technique assisted by intelligent fault detection.

\section{References}

1. C. Lanham, Bak. Instr. Com. (2002).

2. S. Nandi, H. Toliyat, X. Li, IEEE Trans. on Ener. Conv., 20 (4) 719-729 (2005)

3. J. Benali, M. Sayadi, F. Fnaiech, B. Morello, N. Zerhouni, In Sci. Tech. Auto. Con. Comp. Eng. (STA),2013 14th International Conference on (pp. 259-264), IEEE (2013)

4. A. Burr, J. Cheatham, Engle. Cli., NJ (1995).

5. J. Spacek, International Conference-Modern Technique and Technologies (2008).

6. L. Huang, Y. Chen, S. Chen, H. Jiang, In Qual. Reli. Risk. Main. Safe. Engi. (ICQR2MSE), 2012 International Conference on (pp. 1015-1021) IEEE (2012)

7. J. H. Shin, H. B. Jun, Jour. Comp. Desi. Engi., 2 119-127 (2015).

8. I. Howard, Defe. Sci. Tech. Orga, Canb. (Australia) (1994).

9. N. Tandon, A. Choudhury, Tribo. intern., 32 (8) 469-480 (1999).

10. A. K. Jardine, D. Lin, D. Banjevic, Mech syst sign proc, 20(7) 1483-1510 (2006).

11. E. Jantunen, Intern. Jour. COMADEM, 9 (3) 24 (2006).

12. J. Halme, P. Anderson, Jour. Eng. Tribo., 224 (4) 377-393 (2010).

13. D. Petersen, C. Howard, N. Sawalhi, A. M. Ahmadi, S. Singh, Mech. Sys. Sig. Proc., 50 139-160 (2015). 
14. V. N. Patel, N. Tandon, R. K. Pandey, Proce. Tech., 14 312-319 (2014).

15. H. Saruhan, S. Sandemir, A. Çiçek, Jour. appl. res. tech.,12 (3) 384-395 (2014).

16. D. P. Jena, S. N. Panigrahi, Measu, 55 39-50 (2014).

17. A. V Dube, L. S. Dhamande, P. G. Kulkarni, Intern. Jour. of sci. tech. res., 2 (4) 149-155 (2013).

18. F. Cong, J. Chen, G. Dong, M, Pecht, Jour. sou. vibr., 332 (8) 2081-2097 (2013).

19. V. N. Patel, N. Tandon, R. K. Pandey, Proce. Eng., 64, 1582-1591 (2013).

20. J. Mathew, R. J. Alfredson, Jour. Vibr. Acous., 106 (3) 447-453 (1984).

21. N. S. Swansson, S. C. Favaloro, Aero. Res. Lab. Mel. (Australia). (1984).

22. A. M. Al-Ghamd, D. Mba, Mech. Sys. Sig. Proc., 20 (7) 1537-1571 (2006).

23. S. Al-Dossary, R. R. Hamzah, D. Mba, Appl. acous., 70 (1) 58-81 (2009).

24. B. S. Kim, D. S. Gu, J. G. Kim, Y. C. Kim, B. K. Choi, In Eng. Asset Life. Manag. (pp. 596-602). Springer London (2010).

25. B. Kilundu, X. Chiementin, J. Duez, D. Mba, Mech. Sys. Sig. Proc., 25 (6) 2061-2072 (2011).

26. F. Hemmati, W. Orfali, M. S. Gadala, Rolling element bearing condition monitoring using acoustic emission technique (2012).

27. D. H. Pandya, S. H. Upadhyay, S. P. Harsha, Exp. Sys. with Appl., 40 (10) 4137-4145. (2013).

28. J. L. F Chacon, V Kappatos, W. Balachandran, T. H. Gan, App. Acous., 89 88-100 (2015).

29. Z. Peng, N. Kessissoglou, Wear, 255 (7) 1221-1232 (2003).

30. T. J. Harvey, R. J. K. Wood, H. E. G. Wear, 263 (7) 1492-1501 (2007).

31. L. Wang, Y. Yan, Y. Hu, X. Qian, Mea. Appl. (ICSIMA), 2013 IEEE International Conference on (pp. 1-4). IEEE (2013).

32. X. Zhang, B. Wang, X. Chen, Know.-Bas. Sys., (2015).

33. M. Yuwono, Y. Qin, J. Zhou, Y. Guo, B. G. Celler, S. W. Su, Eng. Appl. Arti. Int., (2015).

34. M. Unal, M, Onat, M. Demetgul, H. Kucuk, Measu., 58, 187-196 (2014).

35. N. Tandon, A. Choudhury, NDT Inter., 13 (5) 235 (1999).

36. C. Cempel, Jour. Sou. Vibr., 73 (4) 547-561 (1980).

37. C. Cempel, Mech. Sys. Sig. Proc., 2 (2) 135-151 (1988).

38. D. Brie, Mech. Sys. Sig. Proc., 14 (3) 353- 369 (2000).

39. J. Pineyro, A. Klempnow, V. Lescano, Jour. all. comp., 310 (1) 276-279 (2000).

40. J. P. Dron, L. Rasolofondraibe, F. Bolaers, Inter. Jour. Sol. Stru., 38 (24) 4293-4313 (2001).
41. J. Altmann, J. Mathew, Mech. Sys. Sig. Proc., 15 (5) 963-977 (2001).

42. Y. Lei, J. Lin,Z. He, Y. Zi, Mech. Sys. Sig. Proc., 25 (5) 1738-1749 (2011)

43. Y. Wang,M. Liang, Mech. Sys. Sig. Proc., 25 (5) 1750-1764 (2011).

44. D. Wang, W. Peter, K. L. Tsui, Mech. Sys. Sig. Proc., 35 (1) 176-199 (2013).

45. G. Yu, C. Li, J. Zhang, Mech. Sys. Sig. Proc., 41 (1) $155-175$ (2013).

46. Y. Ming, J. Chen, G. Dong, Mech. Sys. Sig. Proc., 25 (5) 1773-1785 (2011).

47. Y. Zhou, J. Chen, G. M. Dong, W. B. Xiao, Z. Y. Wang, Mech. Sys. Sig. Proc., 26 229-243 (2012).

48. G. Dong, J. Chen, Mech. Sys. Sig. Proc., $33212-$ 236 (2012).

49. A. B. Ming, Z. Y. Qin, W. Zhang, F. L. Chu, Mech. Sys. Sig. Proc., 41 (1) 141-154 (2013).

50. G. Dong, J. Chen, F. Zhao, Jour. Sou. Vibr., 339 396-418 (2015).

51. X. Wang, Y. Zi, Z. He, Mech. Sys. Sig. Proc., 25 (1) 285-304 (2011).

52. N. Sawalhi, R. B. Randall, Mech. Sys. Sig. Proc., 25 (3) 846-870 (2011)

53. K. Feng, Z. Jiang, W. He, Q. Qin, Meas., 44 (9) 1582-1591 (2011).

54. W. T. Peter, D. Wang, Mech. Sys. Sig. Proc., 40(2) 499-519 (2013).

55. W. T. Peter, D. Wang, Mech. Sys. Sig. Proc., 40(2) 499-519. (2013).

56. D. Paliwal, A. Choudhury, T. Govardhan, Proc. Mat. Sci., 5 2347-2355 (2014).

57. H. Wang, J. Chen, G. Dong, Mech. Sys. Sig. Proc., 48 (1) 103-119 (2014).

58. W. He, Y. Zi, B. Chen, F. Wu, F, He, Z, Mech. Sys. Sig. Proc., 54, 457-480 (2015).

59. W. Guo, W. T. Peter, A. Djordjevich, Measu., 45 (5) 1308-1322 (2012).

60. W. C. Tsao, Y. F. Li, D. Du Le, M. C. Pan, Measu., 45 (6) 1489-1498 (2012)

61. S. Zhao, L. Liang, G. Xu, J. Wang, W. Zhang, Mech. Sys. Sig. Proc., 40 (1) 154-177 (2013).

62. S. G. Mallat, Z. Zhang, Sig. Proc., IEEE Transactions on, 41 (12) 3397-3415 (1993).

63. Akhand Rai, S. H. Upadhyay, Tribio. Intern., (2015). http://dx. doi. org/10. 1016/j. triboint. 2015. 12. 037 